\title{
"THE ROAD GOES UPWARD TOWARDS THE LIGHT": THE TOMBS OF ATUAN DE URSULA K. LE GUIN COMO NOVELA DE FORMACIÓN FEMENINA DE FANTASÍA
}

\author{
Paula Rivera Donoso, Universidad de Chile \\ E-mail: paularivera@ug.uchile.cl
}

Recibido: 25 de Mayo

Aceptado: 25 de Junio

Resumen: Este artículo plantea que The Tombs of Atuan es una novela de formación femenina que se aparta de los códigos tradicionales de la variante realista y hegemónica del subgénero. Esto debido a que presenta dos elementos no miméticos: un laberinto subterráneo como morada de dioses y la aparición de un mago. Ambos complejizan el proceso de crecimiento de la joven Tenar, su protagonista, e inciden en que su destino al final de la novela, pese a no culminar en una madurez plena, sí se abra a posibilidades alentadoras, distintas a las resoluciones fatalistas de la novela de formación femenina realista.

Palabras clave: novela de formación femenina, literatura de fantasía, magia, laberinto, individuación.

\begin{abstract}
This article proposes that The Tombs of Atuan is a female coming of age novel that departs from the conventional codes of its hegemonic version, the realistic one. This derives from the presence of two non-mimetic elements: an underground labyrinth as gods' dwelling and the appearance of a wizard. Both elements complicate the growth process of young Tenar, the protagonist, and influence her destiny. Even when, by the end of the novel, her resolution does not reach full maturity, its positive outcome opens Tenar to encouraging possibilities, quite different from the fatalist outcomes of realistic feminine coming of age novels.

Keywords: female coming of age novel, fantasy literature, magic, labyrinth, individuation.
\end{abstract}




\section{INTRODUCCIÓN}

The Tombs of Atuan es la segunda obra de la saga Earthsea ${ }^{1}$, una serie de novelas de fantasía de la norteamericana Ursula K. Le Guin ambientadas en el mundo secundario de Earthsea [Terramar] $]^{2}$. Al igual que la primera novela, que contaba la historia de formación del joven mago Ged, en esta obra se cuenta la historia de formación de Tenar, una muchacha que es sustraída de su familia de origen, despojada de su nombre (reemplazado por el título de Arha: la Devorada) y elegida como la más importante sacerdotisa de una secta consagrada a los dioses Sin Nombre. La infancia y juventud de Tenar transcurren en el aislamiento de tal secta en la isla de Atuan mientras se forma como sacerdotisa. Sin embargo, su llegada a la adolescencia resulta crucial como punto de inflexión en su vida, pues se le presenta a la joven el dominio exclusivo del Laberinto subterráneo de Atuan, un espacio en el que no solo se aprecian las circunvalaciones propias de esta estructura, sino también una penetrante oscuridad que le impide orientarse más que por la memoria. Sumida en este reino de tinieblas, la vida de Tenar cambia cuando conoce al mago Ged, quien, al actuar como mentor y compañero de la joven, le presenta la posibilidad de un mundo nuevo, marcado por la libertad.

En relación con este planteamiento argumental, este artículo propone, a través de la metodología del close reading, que The Tombs of Atuan es factible de ser leída como una novela de formación femenina que se aparta de los códigos tradicionales de la variante realista y hegemónica de este subgénero. Esto debido a que, en su desarrollo, se presentan dos elementos no miméticos que complejizan de manera singular el proceso de crecimiento de su protagonista y que inciden en que su destino al final de la novela, pese a no culminar en una madurez plena, sí se abra a posibilidades alentadoras, de mayor complejidad que las habituales resoluciones fatalistas de la novela de formación femenina realista.

El primero de estos elementos corresponde al Laberinto de Atuan, emblema de constricción social y espiritual y morada concreta de los amenazantes dioses Sin Nombre. En medio del castrante sistema teocrático en el que está prisionera Tenar, el Laberinto es una estructura física y simbólica que anula y limita la expresión de su individualidad. El segundo de estos elementos, por su parte, corresponde al encuentro de Tenar con el personaje de Ged y la relación de complemento que se establece entre ellos a partir de sus sendos poderes y/o conocimientos mágicos. Este compañerismo asienta un vínculo entre el mundo masculino y el femenino que permite a ambos personajes alcanzar la liberación.

A continuación, se realizará una revisión de las características centrales de la novela de formación femenina y luego se analizará la obra de Le Guin a partir de la identificación

1 La serie de Earthsea está compuesta por las novelas A Wizard of Earthsea (1968), The Tombs of Atuan (1970), The Farthest Shore (1972), Tehanu (1990) y The Other Wind (2001), junto con la antología Tales from Earthsea (2001) y cuentos dispersos. Estos trabajos están compilados en The Books of Earthsea (2018).

2 El concepto de mundo secundario se origina en el ensayo On Fairy-Stories (1947), de J.R.R. Tolkien: "[The storyteller] makes a Secondary World which your mind can enter. Inside it, what he relates its 'true': it accords with the laws of that world. You therefore believe it, while you are, as it were, inside" (2008: 351). El término refiere a aquellos universos ficcionales autónomos y no miméticos, como la Tierra Media, Narnia o Terramar, que son regidos por un sistema de leyes distintas a las naturales, las que sin embargo entrañan su propia coherencia interna. 
de tres fases relevantes en el desarrollo de Tenar, a fin de determinar de qué manera los dos elementos no miméticos enriquecen la tradición de historias de crecimiento de heroínas ficcionales.

\section{LA NOVELA DE FORMACIÓN FEMENINA: CARACTERÍSTICAS E INTERROGANTES}

Para comenzar, es necesario remitir a lo que se suele entender por el género de novela de formación, o Bildungsroman, en sus orígenes alemanes:

[Estas son] novelas cuyo tema principal es la representación literaria de las experiencias de un joven protagonista, desde su niñez o adolescencia hasta su madurez, en un proceso de aprendizaje cuya finalidad es lograr la consolidación de la personalidad del individuo y su integración en la sociedad. Asimismo este proceso formativo del protagonista evidencia el conflicto entre sus deseos y los intereses contrarios de la sociedad (Gómez 2009: 108).

Sin embargo, estas características generales presentan matices en el caso de las narraciones centradas en la formación de personajes femeninos, dadas las diferencias socioculturales presentes en los procesos formativos entre hombres y mujeres. De manera sintética, Lagos señala que la principal diferencia es un movimiento opuesto entre el progreso de protagonistas masculinos y femeninos a lo largo de sus sendas obras: "Mientras el héroe crece, madura y se desarrolla, la heroína presenta un desarrollo regresivo" (1996: 35). Esto se presenta, principalmente, debido a que el protagonista masculino suele crecer para incorporarse a la sociedad como un sujeto íntegro, desde un potencial ya maduro. Pero, para la protagonista femenina, muchas veces la madurez significa abjurar de su individualidad y en cambio adoptar la sumisión y la obediencia (a la propia sociedad o al orden masculino, a través del matrimonio) para sobrevivir, lo que suele hacerla desembocar en la desilusión (Lagos 1996: 34-35).

Esto parece deberse, justamente, al énfasis en el conflicto que sostienen los personajes femeninos con la sociedad a la que pertenecen, por lo general de trazas patriarcales (Gómez 2009: 110). Gómez apunta como características específicas de la novela de formación femenina la alternancia de puntos de vista, la presencia de protagonistas sensibles o rebeldes, con tendencia a fantasear con realidades distintas a las que viven, la lucha contra la autocensura y una relación conflictiva con la familia, que transmite códigos de conducta prescritos por el orden social. Sin embargo, como se mencionó anteriormente, en muchas novelas de formación femenina estos rasgos, de matiz subversivo, se ven finalmente anulados en la resolución de la obra. Si bien Lagos señala que esto se debía al deseo de imponer un modelo de conducta a las mujeres en los siglos XVIII y XIX, la alternativa disidente que ella destaca, de predominante autoría femenina, no parece muy alentadora: la protagonista alienada, que rompe con este orden social a través de la locura, el suicidio, la enfermedad o la muerte (1996: 36). ¿Acaso no es posible una novela de formación femenina en la que se imaginen conclusiones más positivas para el desarrollo e integración de la mujer como sujeto, y en las que sus interacciones con el sujeto masculino sean de complemento mutuo en lugar de sometimiento? 
En este punto es conveniente remarcar que la revisión conceptual anterior se concentra sobre todo en novelas realistas, por lo que cabría preguntarse en qué medida una novela de formación perteneciente a la tradición de la literatura no mimética presenta sus propias particularidades. En efecto, como señala Joannou, existe ya una serie de autoras contemporáneas (Doris Lessing, Angela Carter o Jeannete Winterson, por ejemplo) que han usado los recursos de la ficción imaginativa para apartarse de diversas convenciones de la novela de formación masculina, incluyendo la predominancia del realismo (2019: 200). Para Joannou, de hecho, los modelos clásicos de Bildungsroman, masculinos y femeninos, son susceptibles de ser concebidos como conservadores y reintegrativos en sus proyectos ideológicos de homogeneización normativa. Por ello, la académica se centra en destacar novelas de formación femeninas contemporáneas que ofrecen "sophisticated models of individual, interiorised personal development, bringing marginal voices, lesbian as well as heterosexual, black as well as white, to the centre and have consistently accentuated individuality rather than normalcy" (2019: 216). Si bien la novela de formación femenina de fantasía no presentaría subversiones tan explícitas al operar desde el simbolismo y lo no mimético, cabría identificar su genealogía en el contexto de estas otras Bildungsroman.

En la literatura de fantasía, una estructura narrativa habitual para dar cuenta de la formación de sus protagonistas es el monomito, a partir de la secuenciación de patrones míticos propuestos por Joseph Cambpell en su obra The Hero of a Thousand Faces (1949). Sin embargo, como en las novelas de formación realistas, se ha establecido que esta secuencia está planteada principalmente para personajes masculinos (Sobat 1996: 24). Los femeninos, en cambio, suelen poseer otro tipo de experiencias formativas que no calzan de manera tan exacta o clara con los estadios propuestos por Joseph Campbell ${ }^{3}$. Aunque podría pensarse que esto no debiera suponer mayor reflexión, puesto que ambos tipos de héroes emprenden igualmente un viaje de crecimiento homólogo, como indica Lori Campbell, "the obstacles [the female protagonist] must overcome for her journey to be recognized as heroic are different, and, like it or not, negotiating differences must be taken into account as inherent to her journey" (2014: 7).

Estas peculiaridades se vuelven de particular importancia en el contexto de la literatura de fantasía debido a que esta, como señala Attebery, posee además el potencial de trascender las limitaciones socioculturales verídicas que las ficciones realistas procuran reflejar, aunque sea desde la crítica (1992: 91). Aun más: para el teórico norteamericano, la novela de formación femenina realista del siglo XVIII y XIX, con su tendencia a priorizar destinos catastróficos en sus protagonistas, tendría poco que ofrecer a las lectoras en cuanto a transiciones efectivas hacia la maduración (1992: 91). Claramente, se podría añadir, el tejido imaginativo de la fantasía entrañaría la posibilidad de narrar nuevas y originales maneras de crecer como mujer, que puedan servir de orientación, retrospectiva o discusión simbólicas en relación con el proceso formativo de cada lectora, o con las visiones personales que cada una pueda tener de él.

3 Sobat traza un paralelo entre seis estadios de Joseph Campbell para el héroe y cinco de Annis Pratt, en su obra Archetypal Patterns in Women's Fiction (1981), para la heroína. Aunque ambos poseen fases equivalentes, Sobat señala que Pratt considera que las heroínas no logran reintegrarse por completo a una sociedad tradicional al final de su recorrido (1996: 24). 
En los apartados siguientes se procederá con la lectura detallada del proceso de crecimiento de Tenar, a fin de esclarecer de qué manera son justamente las propiedades no miméticas de la fantasía las que inciden en la peculiar formación de la protagonista.

\section{LA MUJER DORMIDA O DEVORADA: TENAR (ARHA) EN EL LABERINTO}

Uno de los primeros elementos del Bildungsroman femenino, el estadio previo del personaje antes de comenzar su proceso de formación, entronca con el cuento de hadas. Según Sobat, la primera sección de The Tombs of Atuan, correspondiente a la vida de Tenar como sacerdotisa consagrada a las tumbas subterráneas, puede leerse como un período de sueño de la protagonista, equivalente al de algunas princesas en los cuentos de hadas (Blancanieves o La Bella Durmiente, por ejemplo) (1996: 24). Sin embargo, a diferencia de este tipo de narraciones, que no abordan la interioridad de sus personajes femeninos en este estado, la novela de Le Guin desarrolla en detalle lo que vive, piensa y siente Tenar durante este proceso larvario. Esto permite ahondar con más profundidad en la transformación de Tenar en una mujer íntegra: en lugar de delegar su despertar exclusivamente a la responsabilidad del personaje masculino (el príncipe), la obra plantea en cambio una complementariedad entre ella como personaje femenino y Ged como masculino, lo que ayudará a ambos en sus sendos procesos de crecimiento.

Pero, antes de la aparición de Ged en la historia, Tenar solo cuenta con sus dominios del Laberinto como medio de conocimiento del mundo. Es necesario recordar que el laberinto es una expresión arquetípica de gran relevancia en el mito y en la literatura, principalmente por las significaciones metafísicas que conlleva su estructura física en tanto objeto material ${ }^{4}$. $\mathrm{Al}$ respecto, se hace indispensable retomar el matiz que subyace a los conceptos de maze y labyrinth ${ }^{5}$. Según señalan Douglas \& Byrne a partir de su rastreo bibliográfico sobre estos conceptos, ambos apuntan a una construcción espacial diseñada de manera intrincada para extraviar al viajero, si bien el labyrinth además apunta a nociones como el regreso al Paraíso (expresado en la llegada al centro del laberinto), desafíos o ritos de paso, iniciación espiritual o las propias dificultades de la vida rumbo a su plenitud (2014: 5). Adicionalmente, su configuración enrevesada no solo pretendería desorientar al explorador, sino también, para resguardar sus tesoros, causar la perdición de ladrones o profanadores que osaran recorrer sus senderos. Por último, existiría una asociación entre sus atributos y los genitales

4 Si bien el laberinto es una figura simbólica habitual en la literatura fantástica, su tratamiento suele diferir del de la literatura de fantasía, tal y como corresponde al hecho de que ambas literaturas sean estéticas distintas, usualmente confundidas. Por esta razón, no se realizará un estado del arte del laberinto fantástico, pues no se considera pertinente para este artículo. Solo se mencionará que este suele expresarse como una alegoría del proceso creativo o intelectual. El laberinto de la fantasía, en cambio, posee un tratamiento más concreto, de modo que su simbolismo se presenta en relación con las propiedades sicológicas de los personajes o con las de los mundos secundarios, como se apreciará a lo largo de este análisis.

5 Se ha considerado pertinente referir a este matiz, inexistente en castellano, porque el idioma original de la obra literaria con la que se está trabajando es el inglés. De hecho, este alcance se pierde en la traducción de la novela para el sello Minotauro (posteriormente propiedad de la editorial Planeta), a cargo de Matilde Horne. 
femeninos, lo que vincularía la noción de la muerte (lo subterráneo) con la vida (la matriz de la mujer como origen o nacimiento) (2014: 6-7).

Así, el Laberinto (Labyrinth) de Atuan enseguida encauza su interpretación hacia derroteros simbólicos vinculados al destino de su supuesta ama: “Le Guin's labyrinth, like her caves, are places that resonates with the subtely and misticism of female power, but Le Guin adds to this the connotation of getting lost and finding one's way, which are associated with labyrinths and mazes, as metaphors for self-transformation" (Douglas \& Byrne 2014: 9).

Esto parece condecirse con la ambigua vida de Tenar en Atuan, pues la joven adquiere el rol de la mujer más poderosa de la zona. Como tal, la muchacha asume con orgullo las profundidades del Laberinto como sus legítimos dominios, que poco a poco va conociendo bajo las rígidas enseñanzas de las sacerdotisas mayores. Esto se ve reflejado en las descripciones del Laberinto, a través de términos que construyen un campo semántico asociado a la oscuridad, la negrura y la opresión:

The throne on its high platform seemed to be curtained on each side with great webs of blackness dropping from the gloom of the roof; whether these were curtains, or only denser shadows, the eye could not make certain. The throne itself was black, with a dull glimmer of precious stones or gold on the arms and back, and it was huge (Le Guin 1993: 177).

Estas descripciones físicas se ven complementadas por descripciones de atmósfera, centradas en las sensaciones que experimenta Tenar al creer apropiarse sicológicamente de este entorno. Así, su concepción original de la oscuridad laberíntica, marcada por la asfixia y el temor al extravío perpetuo, cambia hasta hacerle ver el Laberinto como un espacio que conlleva su propia belleza: "It [The blackness] seemed sweet and peaceful as a starless night, silent, without sight, or light, or life. She plunged into the clean darkness, hurried forward through it like a swimmer through water" (1993: 203).

Sin embargo, en su calidad de novela de formación femenina, este estado inicial de Tenar en The Tombs of Atuan está lejos de suponer una conciliación armónica con los sistemas de valores ofrecidos por su entorno sociocultural. En otras palabras, no puede entenderse este presunto empoderamiento como la forja de la identidad de Tenar, pues la narrativa religiosa que le ha sido impuesta asigna estos progresos a la identidad preexistente de Arha: "She was the First Priestess. All human beings were forever reborn, but only she, Arha, was reborn forever as herself. A hundred times she had learned the ways and turnings of the Labyrinth and had come to the hidden room at last" (1993: 214). Esta posición le permite plantear a Cummins que Tenar, como corresponde a una protagonista de una novela de formación femenina, se encuentra inicialmente en un confinamiento múltiple:

Psychologically her development is arrested between being a child and becoming a woman. Politically she is trapped into carrying out the bidding of the monarch and his religious representative at Atuan, although ostensibly she holds the position of supreme power. Socially she is trapped in the identity of the One Priestess [...] (1993: 42).

Podría proponerse que, en el contexto de esta fase inicial, la joven Tenar ha sido anulada por la sacerdotisa Arha. Sin embargo, el prólogo de la obra ha presentado su nombre original junto con un retazo de su antigua vida familiar; esto, junto con la inserción progresiva 
de inconformidad, anticipan que uno de los temas centrales de la novela estribará en la recuperación de la verdadera identidad de Tenar y su distancia de aquel mundo en el que vive confinada, tal y como corresponde a una heroína de novela de formación femenina.

Uno de estos indicios de inconformidad, que corresponden a una manifestación de insurrección propia de la protagonista de la novela de formación femenina ante su contexto, es la sensación de hastío que siente Tenar ante la reverencia vacua de sus pares: "They all bowed the knee to her now [...]. All spoke to her with elaborate deference. But nothing had changed. Nothing happened. Once the ceremonies of her consecration were over, the days went on as they had always gone" (Le Guin 1993: 194-195). Otro indicio, esta vez vinculado a la capacidad de fantasear con realidades distintas, es el cambio que opera en ella cuando su única amiga, la novicia Penthe, le confiesa su falta de fe en los dioses. Estas palabras le hacen pensar por primera vez a Tenar en un mundo distinto: "[...] she felt as if she had looked up and suddenly seen a whole new planet hanging huge and populous right outside the window, an entirely strange world, one in which the gods did not matter" (1993: 209).

Este estado intermedio de Tenar, entre la prisión física y simbólica del Laberinto y la idea brumosa de otra existencia posible, le permitirá a la protagonista abrirse a la experiencia de cuestionamiento que desencadenará la llegada de Ged.

\section{LA MUJER EN DUERMEVELA, O UNA LUZ EN LAS TINIEBLAS: TENAR ENCUENTRA A GED}

La irrupción de Ged en el universo de las tumbas podría considerarse como un punto de inflexión positiva en la vida de Tenar y en la obra, y constituye un elemento aparentemente impensable en la vertiente hegemónica de la novela de formación femenina realista, en la que los hombres asumen roles patriarcales que atentan contra la individuación de la mujer. Acaso por lo anterior, parte importante de la bibliografía crítica de The Tombs of Atuan se ha centrado en el análisis de la relación entre Tenar y Ged, intentando esclarecer hasta qué punto la liberación de la protagonista depende positiva o negativamente de su vínculo con el mago, una discusión que suele abordarse desde distintas lecturas feministas. Al respecto, pueden identificarse tres corrientes interpretativas principales: una que valora el desarrollo de Tenar por considerar que este se ve propiciado desde un compañerismo junguiano, a partir del complemento entre los principios arquetípicos de lo masculino (animus) y de lo femenino (anima) (Cummins 2014; Douglas \& Byrne 2014; St. John 2006) ${ }^{6}$; otra que desestima el desarrollo de Tenar por considerarlo dependiente de Ged (Molson 1979 y Thompson 1986, ctds. en St. John 2006) ${ }^{7}$; y otra en que, a pesar de encontrar reparos en el

6 Un aspecto en común de estos estudios es que suelen concentrarse en The Tombs of Atuan para sus análisis. En algunos casos, debido al rango de años entre la publicación de la segunda y la cuarta novela, esto se debe a que Tenar solo aparecía en la obra aquí analizada. La posterior publicación de Tehanu permitió que, incluso enfocándose solo en The Tombs of Atuan, el análisis de Tenar contemplara también la faceta madura del personaje.

7 Al respecto, St. John (2006) destaca de estas lecturas argumentos que pueden sintetizarse señalando que Tenar exhibe una pasividad excesiva, y que su mayor importancia como personaje es su rol como guía en el monomito de Ged. 
desarrollo de Tenar, se lo valora como anticipo del crecimiento que posteriormente exhibirá en la novela Tehanu, cuarta obra de la serie de Earthsea ${ }^{8}$ (Nodelman 1995; Littlefield 1995).

En una lectura superficial, parece evidente que Ged salva a Tenar al presentarle una forma de vida distinta, devolverle su nombre original y ayudarla a escapar del Laberinto. Incluso, podría pensarse que la novela plantearía una enésima historia de fantasía de tintes patriarcales al proponer un entorno femenino viciado que solo la llegada de un personaje masculino, portador de luz y magia, puede desafiar.

Sin embargo, una lectura más profunda desmonta la rotundidad de estas apreciaciones. En principio, no puede concebirse a Tenar como un apéndice de Ged en la medida en que ella es la protagonista de la obra y que Ged aparece hacia la mitad de la novela, sin que por ello la focalización abandone a la joven. Para Nodelman,

[...] the rhetoric of Tombs, which focuses on Tenar being freed from the false idea of femininity represented by Arha and becoming herself, gives us no choice but to see her as an individual rather than just the nameless expression of the anima she was - as a character, that is, rather than a psychic component. Instead of disguising the pattern, the fact that she is "a person with an individuality of her own" actively conflicts with the possibility that she is merely part of Ged's psyche (1995: 184).

Otro aspecto relevante en Tenar como personaje autónomo es que, como se enunció en la fase inicial, la joven ya exhibía trazas de individualidad que posteriormente serían potenciadas por la llegada de Ged. Esto indica que Ged no cumple el rol de salvador, sino de ayudante o catalizador, una irrupción positiva que mostrará a Tenar otra existencia posible. Como plantea Attebery, por otra parte:

$[\ldots]$ the male who acts as catalyst for the female's transformation is also, in a sense, herself. He represents those impulses toward independent action and self-definition which society insists the young girl suppresses. Since those qualities are culturally defined as masculine, they must enter the story in male guise, but the outcome is the redefinition of the female. By the end, the heroine has grown to encompass his qualities as well as her own "masculine" initiative and "feminine" wisdom (1992: 96).

En lo que respecta a la crítica del matriarcado sectario de The Tombs of Atuan, resulta discutible proponerla como una crítica al mundo femenino, toda vez que la comunidad en la que vive Tenar corresponde más bien a una representación corrupta de lo femenino simbólico, como insinúa Cummins al señalar la influyente presencia de códigos patriarcales en la actitud de las sacerdotisas superiores y sus obsesiones con el poder (1993: 41). De este modo, es a través del jerárquico vínculo con estas sacerdotisas que se aprecia la relación asfixiante, de visos patriarcales, de la protagonista de la novela de formación femenina con su familia.

Así, podría proponerse más bien que el conflicto entre Tenar y el mundo de Atuan estriba en la recuperación de su identidad femenina legítima, en contraposición a la que

8 Estos estudios analizan al personaje en relación con la saga Earthsea de manera íntegra, centrándose igualmente en la evolución de las concepciones sobre lo masculino y femenino en la serie, aspecto que la propia autora explicitó en algunos ensayos. 
le fue asignada: "Arha is then the anima as a distorted representation of femininity that must be discredited, to be replaced by the individual person Tenar" (Nodelman 1995: 184).

En el marco de este análisis, se adscribe principalmente a la primera corriente interpretativa respecto de la relación entre Tenar y Ged, pues se concibe que ambos se complementan a partir de sus respectivos conocimientos. Por otro lado, si bien es importante considerar que el crecimiento de Tenar como sujeto femenino continúa en novelas posteriores en el ciclo (Tehanu y The Other Wind), cuando ya es una mujer madura, el énfasis de este artículo está en esta novela en particular, que sentará las bases para los nuevos conflictos y las nuevas resoluciones del personaje.

Desde la aparición del mago, la joven pretende demostrarle su propia superioridad, pero a la vez se reconoce incapaz de vencerlo. Esta actitud presupone una atracción inicial hacia él, correspondiente a un despertar sexual, que puede explicarse en la medida en que "Ged is wholly different from her in sex, skin color, place of origin, religion" (Cummins 1993: 4445), y Tenar, para entonces, parece predispuesta a adentrarse en la comunión con la otredad ${ }^{9}$.

Respecto del rol de Ged como mago y compañero varón, resulta llamativo que los hechizos que este ejecuta en Atuan no estén orientados al ataque o a la liberación explícita de lo que lo mantiene preso en las tumbas. Antes bien, la magia que proyecta Ged, de manera coherente con su rol como ayudante y compañero de Tenar, tiene que ver con la guía y la inclinación al descubrimiento: por ejemplo, la luz que emplea para orientarse en el Laberinto, primero a solas y luego con la joven, y un acto de ilusionismo en el que viste a Tenar con un ropaje delicado y elegante, en reemplazo de su túnica negra. Aunque podría interpretarse este acto como un gesto seductor, cabría más bien leerlo como una manifestación simbólica de la persona que Ged descubre en las profundidades de Tenar, como él mismo le señala: "You told me to show you something worth seeing. I show you yourself" (Le Guin 1993: 251). Esta confesión puede leerse como una muestra de que la magia en sí misma no es la única vía de liberación; en este contexto, importará más el descubrimiento de la propia identidad de Tenar y la transformación que su recuperación requiere. En efecto, "Although Ged uses magic to hold off the Old Powers and ensure his survival, magic is not instrumental in his escape [...]. Ged is only able to leave the realm of the Powers because of Arha's knowledge of her subterranean world, and because of their mutual trust" (St. John 2006).

Para St. John, uno de los principales valores de la relación entre Tenar y Ged es, precisamente, el de la confianza mutua, que van desarrollando a lo largo de diversas conversaciones en las que Tenar termina por asumir su deseo de vivir de manera diferente. Esta lectura revela que ambos personajes suponen un peligro para el otro. Tenar tiene el poder de delatar la presencia de Ged, o dejarlo morir, como ha hecho con otros prisioneros. Ged, por su parte, tiene el poder de manipularla con su magia, pues conoce su nombre verdadero y eso, en la tradición de Earthsea, implica obtener un control total del individuo. Sin embargo, ambos eligen confiar en el otro. Esto se aprecia en numerosos gestos, como los engaños que la joven realiza a su superior para ocultar que mantiene con vida a Ged, y

9 Aunque Ged es para entonces un mago célibe, podría señalarse que él también manifiesta una atracción por la muchacha. Destaca el adverbio "softly", que aparece constantemente en la narración durante las veces en que él le habla a ella. Este atributo se encontraba ausente en $A$ Wizard of Earthsea, la primera novela de la saga. De todos modos, Ged solo se unirá sexualmente a Tenar en Tehanu, cuando ambos son ya mayores. 
el gesto del mago de revelarle su propio nombre. Esta simbiosis tiene su máxima expresión en el hallazgo conjunto de parte del anillo mágico de Erreth-Akbé en las tumbas, cuya otra mitad poseía el mago: "The symbol of the bond is the rejoined ring; it reveals the BondRune needed by a king to bring unity to Earthsea. Tenar's rune, then, symbolizes unity; the ring, Tenar's self, the islands of Earthsea are all joined" (Cummins 1993: 46).

Ged manifiesta su rol como ayudante contribuyendo a que Tenar pueda atisbar tanto la mujer en potencia que esconde en su interior como el mundo que le espera más allá de Atuan. Aun así, sobre todo en lo que respecta a la manera en que ambos unen sus conocimientos para huir del Laberinto y los Sin Nombre, una amenaza que se cierne sobre los dos por igual, es factible proponer que ambos son personajes equivalentes. Incluso podría proponerse que, en términos simbólicos, Tenar es tan anima para Ged como Ged es animus para Tenar; ambos se complementan desde aquello que carecían inicialmente: Tenar posee el conocimiento del Laberinto, mientras que Ged posee la magia de la luz para orientar su camino.

\section{LA MUJER DESPIERTA, PERO DEBILITADA: EL VIAJE DE TENAR HACIA EL MUNDO DE EARTHSEA Y HACIA SÍ MISMA}

Sin embargo, el escape del Laberinto no supone la liberación plena de Tenar, quien yace aún lastimada por la carga que su rol como sacerdotisa le ha depositado. Por ejemplo, la joven se aterra del aspecto de Ged al verlo bajo la luz de la superficie (Le Guin 1993: 280). Este rechazo inicial simboliza la dificultad que supondrá para Tenar comenzar a explorar el mundo exterior, a pesar de sus sinceros anhelos de liberación. Por otra parte, Tenar siente un repentino impulso de asesinar a Ged mientras ambos navegan juntos, hasta que el mago vuelve a pronunciar su nombre verdadero y a recordarle su naciente vínculo (1993: 294). Esta pulsión irracional puede entenderse al considerar que la muchacha aún está profundamente herida por su experiencia en Atuan, y que aun cuando los Sin Nombre hayan perecido en el derrumbe del Laberinto, su maldad puede seguir actuando a través de ella si se lo permite. Esta condición de vulnerabilidad aunada a su reciente liberación se ve expresada a través de la metáfora del camino ascendente hacia la luz como paralelo de su escape del Laberinto; en el fondo, la existencia humana, como la joven Tenar ha comprendido, será siempre un viaje en las penumbras hacia la búsqueda de esa luz huidiza:

\footnotetext{
She wept in pain, because she was free. What she had begun to learn was the weight of liberty. Freedom is a heavy load, a great and strange burden for the spirit to undertake. It is not easy. It is not a gift given, but a choice made, and the choice may be a hard one. The road goes upward towards the light; but the laden traveller may never reach the end of it (1993: 295).
}

Tras el derrumbe del Laberinto, por consiguiente, Tenar ha recuperado así su nombre y libertad, pero no posee nada más. En un sentido simbólico, su transformación hacia el final de la novela parece presentarse como un renacimiento, un estado primigenio que recoge tanto la liberación del espíritu como su indefensión, que precede a todo proceso de conformación de la genuina identidad. Podría plantearse entonces que Tenar, hacia el final de la novela, se ve inmersa en un nuevo laberinto, uno de carácter plenamente espiritual, íntimo, y que en lugar de constreñirla ahora solo pretende encaminarla hacia el centro de sí misma. 
Ahora bien, este estado del personaje en el desenlace es radicalmente distinto al estado en que se encontraba su contraparte masculina, Ged, en el desenlace de su propia novela de formación. Como apunta Littlefield, "Unlike Ged in A Wizard of Earthsea, who can go on to become a mage [...], there is no future, no acceptable role, for Tenar [at the end of The Tombs of Atuan]" (1995: 249). En efecto, un contraste entre ambos procesos permite plantear que la formación masculina es más acotada y directa. La historia de Ged en la primera novela de la saga puede leerse de manera cerrada, pues concluye con una clara victoria de crecimiento, mientras que la femenina sería más extensa y ambigua, pues la historia de Tenar en la segunda novela de la saga entrega un desenlace en el que la protagonista apenas alcanza un estado mínimo para comenzar a individuarse. En otras palabras, "for Arha [...], the problem is unleashing rather than mastering herself" (Attebery 1992, 88-89). Ese dominio de sí misma, esa individuación, es algo que se insinúa como un proceso más allá de las páginas de la novela.

Sin embargo, fuera de contar con un proceso formativo más laborioso, aunque la liberación física y espiritual de Tenar se percibe como algo positivo en el contexto de la novela, es el tejido sociocultural mismo de Earthsea, como mundo secundario, el que plantea nuevas interrogantes. La principal podría sintetizarse en la siguiente sentencia de Littlefield: "Le Guin has created an independent woman who awakens to a world that has no place for her" (1995: 250). Pero es la propia autora la que comprende esta complicación. Como explica en su ensayo "Earthsea Revisioned":

Tenar, a heroine, is not a free agent. She is trapped in her situation. And when the hero comes, she becomes complementary to him. She cannot get free of the Tombs without him.

But — a fact some critics ignore - neither can Ged get free without her. They are interdependent. I redefined my hero by making him dependent, not autonomous. But heroines are always dependent, not autonomous - even a Fidelio. They act only with and for their man. I had reimagined the man's role, but not the woman's. I had not yet thought what a female hero might be (Le Guin 2018: 983).

La autora logra resolver este problema, que podría ser equivalente al de la novela de formación femenina realista prototípica en cuanto a la imposibilidad final de la mujer para individuarse, recurriendo a las particularidades de las obras de fantasía. Así, al continuar explorando las temáticas sobre el rol de los hombres y las mujeres en su mundo secundario a lo largo de las novelas siguientes de la saga, sobre todo en lo que refiere a la naturaleza y los roles de género asociados a la magia, Le Guin consigue al fin problematizar estos aspectos y crear un espacio físico y espiritual en donde Tenar podrá continuar aquel desarrollo presentado al final de The Tombs of Atuan.

Como confiesa la autora en su ensayo,

I couldn't continue my hero-tale until I had, as woman and artist, wrestled with the angels of the feminist consciousness. It took me a long time to get their blessing. From 1972 on, I knew there should be a fourth book of Earthsea, but it was sixteen years before I could write it.

The fourth book, Tehanu, takes up where the trilogy left off [...], but now, [...] the world is seen through a woman's eyes. This time the gendering of the point of view is neither hidden nor denied. In Adrienne Rich's invaluable word, I had "revisioned” Earthsea (2018: 984). 
Considerando que en Tehanu Tenar actúa como una figura mentora de una niña ultrajada, mitad humana y mitad dragón, puede entenderse que ahora la protagonista de The Tombs of Atuan ha alcanzado al fin un estado de plena maduración. Uno en el que la mujer puede acompañar, desde experiencias marcadas por su género, a una niña necesitada de apoyo, a fin de que esta pueda desarrollar el máximo potencial de su identidad: convertirse en un dragón.

\section{CONCLUSIONES}

A partir de este estudio, puede concluirse que The Tombs of Atuan efectivamente es factible de ser leída como una novela de formación femenina, pues el proceso de crecimiento de Tenar se condice con los conflictos de las protagonistas de estas obras por desafiar sus contextos socioculturales en miras a su libertad e individuación. Sin embargo, el hecho de que esta sea una novela de formación de fantasía afecta significativamente tanto este desarrollo como su resolución, apartándola de los códigos tradicionales de su variante realista, la más estudiada. Así, la estructura del Laberinto supone una amenaza tanto física como simbólica, que inicialmente se le presenta a Tenar como un falso dominio desde la identidad impostada de Arha, hasta que el personaje logra renunciar a su tentación y escapar de la influencia de los Sin Nombre, recuperando su nombre verdadero y, con ello, la posibilidad de recuperar también su identidad arrebatada. Por otro lado, Ged, como personaje masculino, no cumple el rol castrador de una figura patriarcal, sino de catalizador o complemento para el desarrollo de Tenar. Aunque sus facultades mágicas podrían interpretarse como una guía jerárquica y patriarcal por su sentido simbólico (la luz masculina que ilumina las profundidades femeninas), la novela enfatiza en la naturaleza maligna de las tumbas y el Laberinto, a modo de poder femenino torcido desde el culto de la secta a los Sin Nombre, así como en el hecho de que Tenar y Ged necesitan combinar sus conocimientos (el mundo femenino y el masculino) para salvarse.

Por último, si bien Tenar no logra emerger como un sujeto plenamente formado tras las experiencias de la novela, se evidencia que está en una mejor posición que muchas de sus pares de la novela de formación realista tradicional, lo que le permitirá volverse ella misma mentora de una niña en una novela posterior de la saga. En ese sentido, podría plantearse que la construcción de un universo mimético impondría un corsé narrativo de difícil negociación con los procesos de la heroína, debido a la histórica marginación de la mujer en la sociedad. En cambio, la construcción de un mundo secundario no mimético como Earthsea facilitaría estos procesos de discusión o cuestionamientos, pues en última instancia el tejido social de su narrativa depende de la inventiva de su autor, y en este reside la posibilidad de modificarlo a fin de permitir el desarrollo de sus heroínas, como hizo Le Guin en su creación para continuar ahondando en la formación de Tenar.

A modo de proyección, las conclusiones anteriores permiten plantear que las particularidades estéticas y narrativas de la literatura de fantasía pueden aportar enriquecedoras dimensiones a la novela de formación femenina, presentando así nuevas e insólitas historias de crecimiento que reflejen, desde la ficción más imaginativa, el siempre difícil proceso de convertirse en una mujer plena. 


\section{REFERENCIAS}

Attebery, B. 1992. "Women's Coming of Age in Fantasy." Strategies of Fantasy. Bloomington: Indiana University Press, 87-104.

Campbell, L. (ED.). 2014. "Introduction." A Quest of Her Own: Essays on the Female Hero in Modern Fantasy. North Carolina: McFarland, 4-14.

Cummins, E. 1993. "Earthsea.” Understanding Ursula K. Le Guin. Revised Edition. South Carolina: University of South Carolina Press, 22-64.

Douglas, L. And Byrne, D. 2014. "Womanspace: The Underground and the Labyrinth in Ursula K. Le Guin's Earthsea Narratives.” Literator 35 (1), 1-8. DOI: http://dx.doi. org/10.4102/lit.v35i1.1070

GómEz, C. 2009. "El Bildungsroman y la novela de formación femenina hispanoamericana contemporánea." Epos: Revista de Filología 25, 107-117. DOI: https://doi.org/10.5944/ epos.25.2009.10609

Joannou, M. 2019. "The Female Bildungsroman in the Twentieth Century." A History of the Bildungsroman. Ed. Sarah Graham. Cambridge: Cambridge University Press, Cambridge, 200-216.

Lagos, M. 1996. En tono mayor: relatos de formación de protagonista femenina en Hispanoamérica. Santiago de Chile: Cuarto Propio.

LE GuIn, U. 1993. "The Tombs of Atuan.” The Earthsea Quartet. London: Penguin Books, 169-300.

LE GuIn, U.2018. “Earthsea Revisioned." The Books of Earthsea: The Complete Illustrated Edition. New York: Simon \& Schuster / Saga Press, 979-993.

LitTlefield, H. 1995. "Unlearning Patriarchy: Ursula Le Guin's Feminist Consciousness in The Tombs of Atuan and Tehanu." Extrapolation 36 (3), 244-258. DOI: https://doi. org/10.3828/extr.1995.36.3.244.

Nodelman, P. 1995. "Reinventing the Past: Gender in Ursula K. Le Guin's Tehanu and the Earthsea 'Trilogy."' Children's Literature 23 (1), 179-201. DOI: https://doi:10.1353/ chl.0.0246.

Sobat, G. 1996. "The Night in Her Own Country: The Heroine's Quest for Self in Ursula K. Le Guin's The Tombs of Atuan." Mythlore: A Journal of J.R.R. Tolkien, C.S. Lewis, Charles Williams, and Mythopoeic Literature 21 (3). https://dc.swosu.edu/mythlore/vol21/iss3/5.

St. John, G. 2006. "Magic, Trust, and Choice in the Escape from Atuan." The Looking Glass: New Perspectives on Children's Literature 10 (3). https://www.lib.latrobe.edu. au/ojs/index.php/tlg/article/view/73/87.

Tolkien, J.R.R. 2008. “On Fairy-Stories.” Tales from the Perilous Realm. London: Harper Collins, 315-400. 